\title{
POSITIVE WEAK RADIAL SOLUTIONS OF NONLINEAR SYSTEMS WITH $p$-LAPLACIAN
}

\author{
SORIN BUDIŞAN
}

Abstract. We study the existence and localization results for radial solutions of systems with $p$-Laplacian. The compression and expansion conditions that are used are related to the first eigenvalue of the $p$-Laplacian.

Mathematics subject classification (2010): 34B18.

Keywords and phrases: $p$-Laplacian, positive solution, boundary value problem, cone, fixed point.

\section{REFERENCES}

[1] C. ARANDA, T. GODOY, Existence and multiplicity of positive solutions for a singular problem associated to the p-Laplacian operator, Electron. J. Differential Equations, 132 (2004), 1-15.

[2] L. ERBE AND M. TANG, Uniqueness theorems for positive radial solutions of quasilinear elliptic equations in a ball, J. Differential Equations, 138, 2 (1997), 351-379.

[3] M. Garcia-Huidobro, R. MANASEVICH AND C. S. YARUR, Behavior of positive radial solutions for quasilinear elliptic equations, Proc. Amer. Math. Soc., 129, 2 (2001), 381-388.

[4] M. KRasnosels KII, Positive solutions of operator equations, Noordhoff, Gröningen, 1964.

[5] S. LiU, Existence of solutions to a superlinear p-Laplacian equation, Electron. J. Differential Equations, 66 (2001), 1-6.

[6] H. Lu, D. O'Regan And R. P. Agarwal, Positive radial solutions for a quasilinear system, Appl. Anal., 85, 4 (2006), 363-371.

[7] D. O'Regan AND R. Precup, Positive solutions of nonlinear system with p-Laplacian on finite and semi-infinite intervals, Positivity, 11 (2007), 537-548.

[8] D. O'Regan And H. WAng, Positive radial solutions for p-Laplacian systems, Aequationes Math., 75 1-2 (2008), 43-50.

[9] R. PRECUP, A vector version of Krasnoselskii's fixed point theorem in cones and positive periodic solutions of nonlinear systems, J. Fixed Point Theory Appl., 2 (2007), 141-151.

[10] M. TANG, Uniqueness and global structure of positive radial solutions for quasilinear elliptic equations, Comm. Partial Differential Equations, 26, 5-6 (2001), 909-938.

[11] F. H. Wong, C. C. Chou, Wei-Cheng Lian and Shiueh-Ling Yu, Non-Existence of Positive Radially Symmetric Solutions for the p-Laplacian Boundary Value Problem on Annular Domains, Funkcial. Ekvac., 51 (2008), 269-279. 